\title{
Impact of Postoperative Septic Complications on Recurrence of Colorectal Cancer
}

\author{
Miroslav Levy, Ludmila Lipska, Ladislav Sojka, Jaromir Simsa, Vladimir Visokai
}

Surgical Department, Thomayer Hospital, First Faculty of Medicine Charles University Prague, Czech Republic

\section{ABSTRACT}

Background: About one third of patients racically resected for colorectal cancer develop during follow-up recurrence.

Materials and methods: There were 1951 patients operated for colorectal cancer in Surgical Department, Thomayer Hospital Prague, from 1997 to 2015. Radical R0 operation underwent $68 \%$ of these patients. Postoperative complications occurred in 457 (34.6\%) patients. Impact of postoperative complications on disease free interval was studied in a prospective study.

Results: We identified minor complications in 90 patients $(6.8 \%)$, moderate complications in 28 patients (2.1\%), anastomotic leakage in 67 patients (5\%) and severe septic complications in 20 patients $(1.5 \%)$. Another 255 patients $(19.3 \%)$ had a different, non-inflammatory complications (pulmonary embolism, bowel obstruction, heart failure, etc.). Significantly worse disease-free interval was found in patients with severe septic complications.

Conclusion: In our cohort of $\mathrm{R} 0$ operated patients, postoperative complication is the second most important prognostic factor following TNM stage of the colorectal cancer. Severe septic complications has an adverse effect on the further course of the disease in terms of relapse. Other potentially septic complications such as anastomotic leakage have no essential impact on recurrence. Therefore, it is necessary to prevent the development of sepsis.

Key words: colorectal cancer, recurrence, septic complications

\section{INTRODUCTION}

Colorectal cancer is the third most common cancer in men $(10.0 \%$ of the total) and the second in women (9.2\% of the total) worldwide. Almost $55 \%$ of the cases occur in more developed regions. Mortality is lower (694,000 deaths, $8.5 \%$ of the total) with more deaths (52\%) in the less developed regions of the world, reflecting a poorer survival in these regions (1). Countries with the highest incidence rates include Australia, New Zealand, Canada, the United States, and parts of Europe. The countries with the lowest risk include China, India, and parts of Africa and South America. Age-world-standardized incidence rate In Czech Republic is $4^{\text {th }}$ world highest, neighboring with $1^{\text {st }}$ and $2^{\text {nd }}$ highest incidence rate. Worldwide mortality attributable to colorectal cancer is approximately half that of the incidence.
Corresponding author:

Miroslav Levy, M.D., Ph.D., MBA

Surgical Department,

Thomayer Hospital

Videnska 800, 14059 Prague 4

Czech Republic

Phone: + 420724864244

E-mail: miroslav.levy@ftn.cz 
The goal of all cancer research and treatment is to prevent people dying from the disease. Knowledge has been accruing rapidly about actions and interventions that could lead to a reduction in death from colorectal cancer by reducing the risk of developing the disease, identifying the disease at a stage when it is more curable, or improving the outcome of treatment (2). In past decades there were considerable therapeutic improvements for colorectal cancer, focusing on improved surgical and diagnostic techniques and new chemotherapy protocols. Postoperative morbidity is decreasing, but despite improvement in surgical technique, bowel preparation, and prophylactic antibiotics, colorectal surgery is associated with a $20 \%$ to $40 \%$ morbidity rate. Complications rate is high particularly in advanced disease, elderly patients and on an emergency setting (3). The aim of this study is to evaluate the impact of postoperative complications following radical surgery for colorectal cancer on disease free interval.

\section{METHODS}

There were 1951 patients operated for colorectal cancer in Surgical Department, $1^{\text {st }}$ Medical Faculty, Thomayer Hospital Prague, from 1997 to 2015 according to our standard. All patients were operated with open approach. In emergency operations of colorectal cancer with curative intent, the same principles of radicality as in elective procedures are followed. Histological specimens were evaluated at the Department of Pathology Thomayer Hospital. Lymph nodes are retrieved by surgeons from the native specimen. Stage of the cancer used in statistical analysis in the study was according to the UICC TNM classification and was based on clinical data, imaging results, operation records and surgical specimens. For $\mathrm{N}$ status description at least
12 lymphonodes must be histologically examined, otherwise stated as NX stage. Radical RO operation underwent $68 \%$ of these patients.

Minimally two year intensive system of follow-up was implemented. Patients were followed up at an interval of three months during first year and six months interval from $2^{\text {nd }}$ to $5^{\text {th }}$ year. Thereafter, the patients were seen yearly. Physical examination, full history, serum carcinoembryonic antigen (CEA), carbohydrate antigen 19-9 (CA 19-9) and abdominal ultrasound were performed at each follow-up visit. CT scan of abdomen and chest X-ray were performed yearly, colonoscopy biennially. If recurrence was suspected MRI or PET/CT scan was performed (table 1).

Postoperative complications were monitored during hospitalisation and recorded to protocol. Several types of complications were diagnosed. Special importance was devoted to septic complication. Thereafter, these complications were divided according to the ClavienDindo classification (4) (table 2). Any type of complication occurred in 452 (39.6\%) patients.

Patients were divided according to the ClavienDindo classification of postoperative complications into three groups : group I - minor complications (ClavienDindo classification I, II), group II - moderate complications (Clavien-Dindo III), group III - anastomotic leakage, group IV - severe septic complications and group V other, non inflammatory complications.

Statistical analysis of the data was performed by using the S.A.S program, version 6.12 and the Statistica program. Descriptive statistics (average, median, standard deviation, maximum, minimum) were calculated for the whole group of patients, as well as for individual subgroups. Comparison of the groups according to different criteria was made with the Wilcoxon non-pair test. The values equal to or less than 0.05 were considered significant.

Table 1 - Follow-up system in Thomayer Hospital

\begin{tabular}{|c|c|c|c|c|c|c|c|c|c|c|c|c|c|c|c|}
\hline \multicolumn{16}{|c|}{ Months after operation } \\
\hline & 3 & 6 & 12 & 18 & 24 & 30 & 36 & 42 & 48 & 54 & 60 & 72 & 84 & $\begin{array}{c}96 \\
120\end{array}$ & $\begin{array}{c}108 \\
144 \\
166\end{array}$ \\
\hline History & $X$ & $X$ & $X$ & $x$ & $X$ & $X$ & $X$ & $X$ & $X$ & $X$ & $X$ & $X$ & $x$ & $X$ & $x$ \\
\hline Physical examination & $X$ & $x$ & $X$ & $x$ & $x$ & $X$ & $x$ & $X$ & $X$ & $X$ & $X$ & $x$ & $x$ & $x$ & $x$ \\
\hline CEA, CA19-9 & $x$ & $X$ & $X$ & $x$ & $x$ & $x$ & $x$ & $x$ & $X$ & $X$ & $X$ & $X$ & $x$ & $X$ & $x$ \\
\hline Ultrasonography & $X$ & $X$ & $X$ & $x$ & $x$ & $X$ & $\mathrm{X}$ & $X$ & $X$ & $X$ & $X$ & $X$ & $X$ & $X$ & $x$ \\
\hline $\mathrm{CT}$ & & & $x$ & & $x$ & & $x$ & & $x$ & & $x$ & $x$ & $x$ & $x$ & $x$ \\
\hline Coloscopy & & & & & $x$ & & & & $x$ & & & $x$ & & $x$ & \\
\hline Chest $X$ ray & & & & & $x$ & & & & $x$ & & & $x$ & & $x$ & \\
\hline
\end{tabular}


Table 2 - Clavien-Dindo classification of surgical complications

\begin{tabular}{ll}
\hline Grades & Definition \\
\hline Grade I: & $\begin{array}{l}\text { Any deviation from the normal postoperative course without the need for pharmacological treatment or surgical, endoscopic and } \\
\text { radiological interventions. }\end{array}$ \\
& $\begin{array}{l}\text { Allowed therapeutic regimens are: drugs as antiemetics, antipyretics, analgetics, diuretics and electrolytes and physiotherapy. This grade } \\
\text { also includes wound infections opened at the bedside. }\end{array}$ \\
& Requiring pharmacological treatment with drugs other than such allowed for grade I complications. \\
& Blood transfusions and total parenteral nutrition are also included. \\
Grade II: & Requiring surgical, endoscopic or radiological intervention \\
\hline Grade III: & intervention not under general anesthesia \\
\hdashline Grade III-b: & intervention under general anesthesia \\
\hline Grade IV: & Life-threatening complication (including CNS complications) $\neq$ requiring IC/ICU-management \\
\hline Grade IV-a: & single organ dysfunction (including dialysis) \\
\hline Grade IV-b: & multi organ dysfunction \\
\hline Grade V: & Death of a patient \\
\hline
\end{tabular}

\section{RESULTS}

In all operated patients (1951) there were $1139 \mathrm{rad}-$ ically resected patients (RO operation) $-58,4 \%$. In 452 patients there were 600 complications recorded. One complication occurred in 341 patients, two in 79, three in 29 , four in one and five complications in two patients (table 3). From RO resected patients for our analysis 150 patients were excluded due to postoperative dead or unclear definition of complication. During follow-up, there were 313 relapses diagnosed, 87 patients from these died.

For statistical analysis all complication were divided into four groups: minor inflammatory complications - 90 patients, moderate inflammatory complications - 28 patients, anastomotic leakage - 67 patients and severe septic complications: - 20 patients. Another 255 patients had a different, non-inflammatory complications (table 4).

Significantly worse 5 year disease-free interval was found in patients with severe septic complications $(p=0,0405)$. Other, less serious septic complications also increase the risk of recurrence, but not statistically significantly (fig. 1, table 5).

\section{DISCUSSION}

The most frequent postoperative surgical complications after colorectal resections are surgical site infection, anastomotic leakage, intraabdominal abscess, ileus and bleeding (5).

The combination of contaminated environment, major surgery and cancer patients is associated with a high incidence of wound infection. In open colorectal surgery the incidence of SSI varies from $2-25 \%(6,7)$.
Table 3 - Postoperative complications

\begin{tabular}{cc}
\hline Number of complications & patients \\
\hline 0 & 687 \\
\hline 1 & 341 \\
\hline 2 & 79 \\
\hline 3 & 29 \\
\hline 5 & 1 \\
\hline
\end{tabular}

Table 4 - Complications according severity

\begin{tabular}{lc}
\hline Complications & patients \\
\hline $\begin{array}{l}\text { Minor inflammatory complications (wound abscess etc.) } \\
\text { Clavien-Dindo I, II }\end{array}$ & 90 \\
\hline Moderate inflammatory complications & \\
(intraabdominal abscess etc.) Clavien-Dindo III & 28 \\
\hline Anastomotic leakage & 67 \\
\hline Severe septic complications (sepsis, peritonitis) & 20 \\
\hline non-inflammatory complications (pulmonary embolism, & \\
bowel obstruction, heart failure, etc.). & 255 \\
\hline
\end{tabular}

Table 5 - Disease free survival in groups of patients

\begin{tabular}{lcc}
\hline Group & DFI 3 year & DFI 5 year \\
\hline No complications & $76,1 \%$ & $68,6 \%$ \\
\hline Other complications (no inflammatory) & $75,3 \%$ & $68,2 \%$ \\
\hline Minor inflammatory complications & $69,3 \%$ & $66,3 \%$ \\
\hline Moderate inflammatory complications & $68 \%$ & $64,8 \%$ \\
\hline Anastomotic leakage & $66,3 \%$ & $64,9 \%$ \\
\hline Severe septic complications & NA & $32,3 \%$ \\
\hline
\end{tabular}

Laparoscopic approach lowers the rate of surgical site infection significantly (8). In our cohort of patients (all open surgery) we found $8 \%$ of wound infections.

An anastomotic leakage is one of the most serious complications [ranges from $2.9 \%$ to as high as $15.3 \%$, in anterior rectal resections up to $24 \%$ (9)] affecting not only morbidity and mortality after resection for colo- 


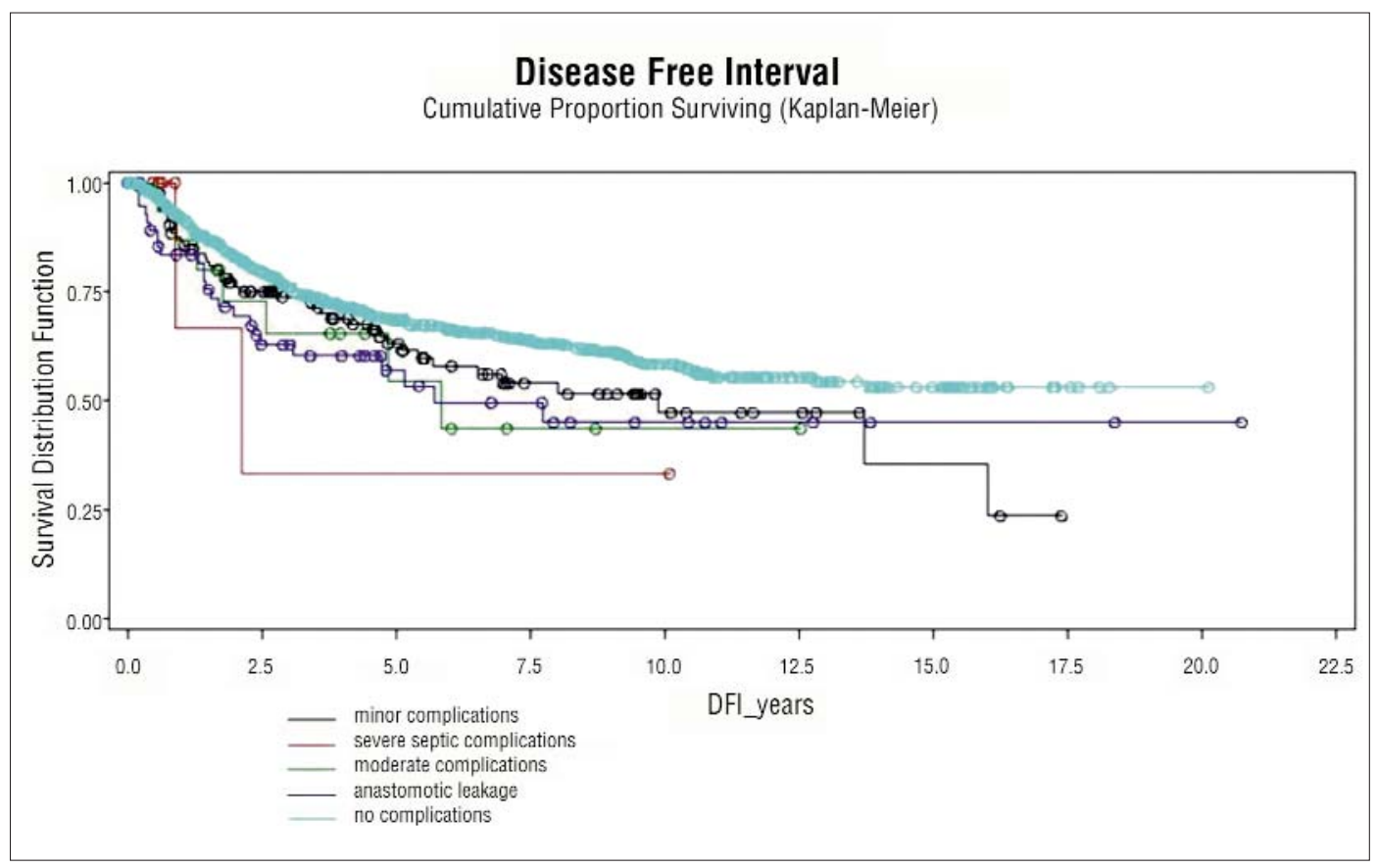

Figure 1 - Disease free interval according to post-operative complications

rectal cancer but also seems to be important factor for local reccurence (10). There were reports, indicating 2,5 times higher risk for local reccurence development after anastomotic leakage in rectal cancer resected patients compared with group without leakage (11). On the contrary Norwegian group in large analysis did not confirm any influence of anastomotic leakage on the local reccurence rate; however, no data on the diseasefree survival were reported (12). Italian study showed that the incidence of local reccurence in patients with anastomotic leakage (24\%) was significantly higher than that in the nonleakage group (11.1\%) (13). Swedish authors confirmed in patients with a perineal infection after an abdominoperineal resection an increased incidence of local recurrence. However, there was no association between abdominal sepsis and prognosis after surgery for rectal cancer (14). In an Australian study on big cohort of patients Walker et al. (15) discussed inflamation-related immunological process that might enhance cancer recurrence.

The demonstration of impact of postoperative complications on oncological result supports the importance of good surgical technique and good perioperative care. In rectal cancer patients there is a question of reduction of the rate of anastomotic leakage by diverting stoma especially in low rectal resections in patients with significant comorbidities, neoadjuvant radiochemotherapy and feculent peritonitis, and also prevention of serious consequences of sepsis associated with anastomotic leakage. In case of anastomotic leakage it is necessary to prevent the development of sepsis, we have to act early when first signs of possible anastomotic leakage occurs. Despite the good results with conservative therapy (including antibiotics), the indication for surgical repair of anastomotic leakage should be made as early as possible to improve patient outcome (5).

The mechanism of progression in septic postoperative course is still unclear. The systematic inflamatory response has been shown to be associated with a poor outcome in patients after curative treatment of colorectal cancer (16). Reason for cancer progression could be in changes in immune system, which are started by sepsis and as a second factor changes in cancer treatment. Patients without complication received adjuvant therapy earlier than in complications group. If complications are serious it may happen that they do not receive it at all.

Sepsis-induced immunosuppression has long been considered a factor in late mortality of patients with sepsis. It seems that regulatory T cells (Tregs) and pro inflammatory citokines should play important role in cancer progression in septic patients. There was found an increase in the percentages of Tregs in peripheral blood circulating CD4+ T cells from patients with sepsis (17). Suppression of tumour-specific CD4+ T cells by 
regulatory $\mathrm{T}$ cells is associated with progression of human colorectal cancer (18).

The question is, how to diagnose potentially septic complication early (before clinical symptomatology). Some study showed that $\mathrm{C}$ reactive protein and/or procalcitonin are useful biochemical markers that could accurately differentiate between infectious and noninfectious complications $(19,20)$.

\section{CONCLUSION}

In conclusion, we showed that severe septic complications has an adverse effect on the further course of the disease in terms of relapse. Therefore, it is necessary to prevent the development of sepsis. In case of inflammatory postoperative complications we we must act quickly, before the onset of sepsis.

\section{Acknowledgments}

The authors thanks all colleagues contributing in colorectal cancer treatment.

\section{Disclosures}

The authors declare no commercial interest.

\section{Role of the funding source}

This work was supported by grants IGA NT134244/2012, GACR P304/12/1585 - covers data analysis, statistical analysis, travel expenses.

\section{Conflict of interest statement}

The authors have declared no conflicts of interest.

\section{REFERENCES}

1. International Agency for Research on Cancer, GLOBOCAN 2012 : Estimated Cancer Incidence, Mortality and Prevalence Worldwide in 2012. http://globocan.iarc.fr/Pages/fact_sheets_cancer.aspx

2. Levy M, Visokai V, Lipska L, Topolcan 0 . Tumor markers in staging and prognosis of colorectal carcinoma. Neoplasma. 2008;55(2):138-42.

3. Alves A, Panis Y, Mathieu P, Mantion G, Kwiatkowski F, Slim K. Postoperative mortality and morbidity in French patients undergoing colorectal surgery: results of a prospective multicenter study. Arch Surg. 2005;140(3):278-283

4. Dindo D., Demartines N., Clavien P.A.; Classification of surgical complications: a new proposal with evaluation in a cohort of 6336 patients and results of a survey. Ann Surg. 2004;240(2):205-13.

5. Kirchhoff $P$, Clavien PA, Hanhloser D. Complications in colorectal surgery: risk factors and preventive strategies. Patient Saf Surg. 2010; 4:5.

6. Blumetti J, Luu M, Sarosi G, Hartless K, McFarlin J, Parker B, et al. Surgical site infections after colorectal surgery: do risk factors vary depending on the type of infection considered? Surgery 2007;142: 704711

7. Tang R, Chen HH, Wang YL, Changchien CR, Chen JS, Hsu KC, et al. Risk factors for surgical site infection after elective resection of the colon and rectum: a single-center prospective study of 2,809 consecutive patients. Ann Surg. 2001;234:181-9

8. Khan MN, Fayyad T, Cecil TD, Moran BJ. Laparoscopic versus open appendectomy: the risk of postoperative infectious complications. JSLS. 2007;11:363-367

9. Platell C, Barwood N, Dorfmann G, Makin G. The incidence of anastomotic leaks in patients undergoing colorectal surgery. Colorectal Dis. 2007:9:71-79

10. Park IJ. Influence of Anastomotic Leakage on Oncological Outcome in Patients with Rectal Cancer. J Gastrointest Surg 2010; 14:1190-1196

11. Branagan G, Finnis D, Wessex Colorectal Cancer Audit Working Group. Prognosis after anastomotic leakage in colorectal surgery. Dis Colon Rectum 2005;48:1021-1026

12. Eriksen MT, Wibe A, Norstein J, Haffner J, Wiig JN, Norwegian Rectal Cancer Group. Anastomotic leakage following routine mesorectal excision for rectal cancer in a national cohort of patients. Colorectal dis 2005;7:51-57

13. Miccini M, Borghese O, Scarpini M, Cassini D, Gregori M, Amore Bonapasta S, et al. Anastomotic leakage and septic complications: impact on local recurrence in surgery of low rectal cancer. Ann Ital Chir. 2011;82(2):117-123.

14. Kressner U, Graf W, Mahteme H, Pțhlman L, Glimelius B. Septic complications and prognosis after surgery for rectal cancer. Dis Colon Rectum. 2002;45(3):316-321.

15. Walker KG, Bell SW, Rickard MJ, Mehanna D, Dent OF, Chapuis PH et al. Anastomotic leakage is predictive of diminished survival after potentially curative resection for colorectal cancer. Ann Surg 2004; 240:255-259

16. Balkwill F, Mantovani A. Inflammation and cancer: Back to Virchow? Lancet 2001;357:539-545

17. Hiraki S, Ono S, Tsujimoto H, Kinoshita M, Takahata R, Miyazaki H, et al. Neutralization of interleukin-10 or transforming growth factor- $\beta$ decreases the percentages of CD4+CD25+ Foxp3+ regulatory T cells in septic mice, thereby leading to an improved survival. Surgery. 2012 ;151(2):313-322.

18. Betts G, Jones E, Junaid S, El-Shanawany T, Scurr M, Mizen P, et al. Suppression of tumour-specific CD4+ T cells by regulatory $T$ cells is associated with progression of human colorectal cancer. Gut. 2012; 61(8):1163-71

19. Oberhofer D, Juras J, Pavičić AM, Žurić IR, Rumenjak V. Comparison of C-reactive protein and procalcitonin as predictors of postoperative infectious complications after elective colorectal surgery. Croat Med J. 2012;53(6): 612-619

20. Ortega-Deballon P, Radais F, Facy 0 , d'Athis P, Masson D, Charles PE, et al. C-reactive protein is an early predictor of septic complications after elective colorectal surgery. World J Surg. 2010;34(4):808-14. 